\section{Aleitamento materno e aspectos nutricionais de crianças da escola de educação infantil de uma instituição hospitalar da cidade de Passo Fundo - RS}

\author{
Breastfeeding and nutritional aspects of children from \\ the school of early childhood education of a hospital \\ institution in the city of Passo Fundo - RS
}

\author{
Patrícia Simioni Vanzin, Graziela de Carli, Daiana Argenta Kümpel
}

\section{RESUMO}

OBJETIVO: verificar as práticas do aleitamento materno e os aspectos nutricionais de crianças da escola de educação infantil de uma instituição hospitalar na cidade de Passo Fundo - RS. MÉTODOS: foi aplicado um questionário de investigação com variáveis sociodemográficas, práticas sobre aleitamento materno, dados maternos, além de um questionário de consumo alimentar para crianças menores de cinco anos e aferição das medidas antropométricas para avaliação do estado nutricional. Os dados foram digitados e analisados em software de estatística, para as variáveis qualitativas foram apresentadas as frequências absoluta e relativa simples e para as variáveis quantitativas foram calculadas as medidas de tendência central e dispersão. Para as análises de associação foi aplicado o teste qui-quadrado considerando o nível de significância de 95\%. RESULTADOS: observou-se neste estudo que a maioria das crianças não recebeu aleitamento materno exclusivo até o sexto mês de vida, conforme recomendação do Ministério da Saúde. Mesmo com prevalência de eutrofia segundo índice de massa corporal/idade, foi constatado que o consumo alimentar das crianças maiores de dois anos em sua maioria encontra-se em risco para futuras complicações quando comparado a menores de dois anos. No entanto foi identificado um fator positivo pelo consumo de frutas, verduras, legumes, carnes e feijão, em ambas as idades. CONSIDERAÇÕES FINAIS: Dessa forma, torna-se fundamental destacar que a influência dos pais em relação à alimentação dos seus filhos deve ser positiva, bem como no ambiente escolar, para que possam juntos atuar como agente transformador nos hábitos alimentares das crianças.

PALAVRAS-CHAVE: Amamentação; Consumo alimentar; Estado nutricional.

\section{ABSTRACT}

OBJECTIVE: to verify the breastfeeding practices and nutritional aspects of children of the preschool of a hospital institution in the city of Passo Fundo - RS. METHODS: a research questionnaire with sociodemographic variables, breastfeeding practices, maternal data, a food consumption questionnaire for children under five years of age and anthropometric measures to assess nutritional status were applied. Data were entered and analyzed using statistical software, for the qualitative variables the simple absolute and relative frequencies were presented and for the quantitative variables the measures of central tendency and dispersion were calculated. For the association analyzes, the chi-square test was applied considering the significance level of 95\%. RESULTS: It was observed in this study that most children did not receive exclusive breastfeeding until the sixth month of life, as recommended by the Ministry of Health. Even with prevalence of eutrophy according to body mass index / age, it was found that food intake Most children over the age of two are at risk for future complications compared to children under two. However, a positive factor was identified by the consumption of fruits, vegetables, meat and beans at both ages. CONCLUSION: Thus, it is essential to emphasize that the influence of parents in relation to their children's eating habits must be positive, as well as in the school environment, so that they can together act as a transforming agent in children's eating habits. 


\section{INTRODUÇÃO}

A Organização Mundial da Saúde (OMS) destaca o aleitamento materno na primeira hora de vida como um item importante para promoção, proteção e suporte à amamentação, além de apresentar baixo custo e de se associar a uma duração prolongada do aleitamento materno ${ }^{1,2}$.

Está comprovado que o leite materno apresenta redução e controle do surgimento de doenças na infância com repercussões na vida adulta dos indivíduos. Esse alimento apresenta vantagens como prevenção de alergias, desenvolvimento psicológico mais consistente, evita problemas respiratórios, melhor absorção dos nutrientes dos alimentos, melhores defesas imunológicas e reduz a mortalidade infantil ${ }^{3}$.

Aos seis meses, deve-se iniciar com a introdução alimentar ou alimentação complementar, onde outros alimentos são oferecidos, além do leite materno ${ }^{4}$. 0 período gestacional conjuntamente com os dois primeiros anos de vida da criança é de suma importância, por tratar-se dos primeiros mil dias, tempo propício em que pode ocorrer a prevenção de doenças crônicas não transmissíveis (DCNT)5.

Um dos problemas que pode ocorrer, como resultado de uma má nutrição é a obesidade infantil, a qual é considerada um problema grave de saúde pública e tem crescido nos últimos anos. Com isso, a publicidade que envolve alimentos infantis deve ser cautelosa, pois forma hábitos que seguem pela vida ${ }^{6}$. 0 aumento do consumo de alimentos ultraprocessados e diminuição do consumo dos alimentos in natura e minimamente processados, justifica-se pela rotina acelerada de jornada de trabalho dos pais, levando à escassez de tempo para preparar uma alimentação mais saudável, levando ao aumento de peso e consumo alimentar excessivo ${ }^{7}$.

A avaliação do estado nutricional tem se tornado um aspecto importante para situações de risco, planejamento alimentar, ações de saúde à criança e prevenções de doenças, assim como a obesidade, diabetes infantil mellitus, risco de hipertensão, entre outras ${ }^{8,9}$.

Sabe-se que o comportamento alimentar na infância deve ser construído a partir dos seis meses através da introdução alimentar, entretanto diversos fatores podem influenciar esta prática por diversos fatores, tais como aspectos fisiológicos e psicológicos da criança, bem como fatores relacionados com a família, mídia, amigos, colegas, escola. Diante do exposto, este estudo teve como objetivo verificar as práticas do aleitamento materno e os aspectos nutricionais de crianças da Escola de Educação Infantil de uma instituição hospitalar na cidade de Passo Fundo - RS.

\section{MÉTODOS}

Trata-se de um estudo de delineamento transversal realizado com pais e crianças matriculadas em creche de uma Instituição Hospitalar, no período de maio a setembro de 2019. 
A amostra foi composta por 72 crianças, de ambos os sexos, matriculadas na creche do Hospital de Clínicas de Passo Fundo - RS. Foram excluídas as crianças com idade superior a quatro anos e as crianças impossibilitadas para a aferição completa das medidas antropométricas e também aquelas a quais os pais não assinaram o Termo de Consentimento Livre e Esclarecido (TCLE).

Para obtenção dos dados foi elaborado um questionário com variáveis demográficas (sexo, faixa etária), dados maternos (faixa etária, estado conjugal) e sobre amamentação da criança (tempo do aleitamento materno, motivo da interrupção). A avaliação da classe econômica foi realizada de acordo com o Critério de Classificação Econômica Brasil (ABEP, 2018), sendo classificado conforme o somatório de pontos em classe econômica A, B, C, D ou E. As questões sobre alimentação foram baseadas no questionário do SISVAN (Sistema de Vigilância Alimentar e Nutricional, 2008) de acordo com a faixa etária da criança, sendo um questionário aplicado para menores de seis meses e outro para crianças acima de dois anos. Para os menores de seis meses, as questões caracterizam a prática do aleitamento materno e identificam o tipo dos alimentos atualmente oferecidos à criança. Já para as crianças entre seis meses e dois anos, investigou-se a alimentação atual da criança e a frequência com que são consumidos determinados alimentos.

Para avaliar o estado nutricional, foram aferidas as medidas antropométricas de peso e altura. Nas crianças menores de 2 anos, utilizou-se balança pediátrica digital e antropômetro de madeira para avaliar peso e comprimento, respectivamente. Nas crianças de 2 a 4 anos, o peso foi verificado em balança digital e a altura com fita métrica. 0 estado nutricional foi classificado pela curva de crescimento, conforme a faixa etária e gênero da criança, através do índice de massa corporal (IMC) para idade (2006). A aferição antropométrica seguiu normas padronizadas9.

Os dados foram digitados e analisados em software de estatística. Para as variáveis qualitativas foram apresentadas as frequências absoluta e relativa simples e para as variáveis quantitativas foram calculadas as medidas de tendência central e dispersão. Para as análises de associação foi aplicado o teste qui-quadrado considerando o nível de significância de 95\%.

Este estudo foi aprovado pelo Comitê de Ética em Pesquisa da Universidade de Passo Fundo, sob parecer 3.281.351. Além disso, todos os participantes da pesquisa foram preservados através do Termo de Consentimento Livre e Esclarecido.

\section{RESULTADOS}

Foram avaliadas 72 crianças, prevalecendo o sexo feminino, faixa etária de 1-2 anos. Em relação às características dos pais elou responsáveis, a maior parte era da faixa etária superior a 30 anos, casados, com escolaridade de 9 anos ou mais, classe econômica B. 
Em relação ao tempo de AME, verificou-se que a maioria não foi amamentada até os seis meses, conforme recomenda a OMS. Quanto aos motivos da interrupção precoce da amamentação, destacou-se leite insuficiente seguido de retorno ao trabalho ou estudo entre outros motivos.

Ao avaliarmos o estado nutricional das crianças, através do IMC/idade, verificamos prevalência de eutrofia em $48,6 \%$, entretanto, nota-se que o excesso de peso também foi relevante em $47,2 \%$, segundo as curvas da OMS, conforme Tabela 1.

Tabela 1. Características sociodemográficas dos pais/responsáveis e estado nutricional de crianças matriculadas em uma creche de uma Instituição Hospitalar de Passo Fundo / RS, 2019. (n=72)

\begin{tabular}{|c|c|c|c|}
\hline Variável & Categoria & $\mathrm{n}$ & $\%$ \\
\hline \multirow[t]{2}{*}{ Sexo } & Masculino & 35 & 48,6 \\
\hline & Feminino & 37 & 51,4 \\
\hline \multirow[t]{2}{*}{ Faixa etária das crianças } & $<2$ anos & 48 & 66,7 \\
\hline & $>2$ anos & 24 & 33,3 \\
\hline \multirow[t]{2}{*}{ Faixa etária* } & $21-30$ anos & 33 & 45,8 \\
\hline & $>30$ anos & 39 & 54,2 \\
\hline \multirow[t]{3}{*}{ Estado conjugal* $^{*}$} & Solteiro & 5 & 6,9 \\
\hline & Casado & 63 & 87,5 \\
\hline & Divorciado & 4 & 5,6 \\
\hline \multirow[t]{3}{*}{ Escolaridade* $^{*}$} & Mais de 12 anos & 34 & 47,2 \\
\hline & 9-12 anos & 34 & 47,2 \\
\hline & Menos de 8 anos & 4 & 5,6 \\
\hline \multirow[t]{3}{*}{ Classe econômica } & $A$ & 8 & 11,1 \\
\hline & B1-B2 & 50 & 69,4 \\
\hline & $\mathrm{C} 1-\mathrm{C} 2$ & 14 & 19,4 \\
\hline \multirow[t]{3}{*}{ Tempo total do $\mathrm{AM}^{\star *}$} & $<6$ meses & 49 & 68,1 \\
\hline & 6-12 meses & 17 & 23,6 \\
\hline & Não mamou no peito & 6 & 8,3 \\
\hline Motivos da interrupção & Leite insuficiente & 22 & 30,6 \\
\hline \multirow[t]{4}{*}{ do $A M E^{* *}$} & Retorno ao trabalho & 17 & 23,6 \\
\hline & Falta de aderência (criança) & 10 & 13,9 \\
\hline & Outro motivo & 16 & 22,3 \\
\hline & Ainda amamenta & 7 & 9,7 \\
\hline \multirow[t]{4}{*}{ Estado nutricional } & Magreza & 3 & 4,2 \\
\hline & Eutrofia & 35 & 48,6 \\
\hline & Excesso de peso & 17 & 23,6 \\
\hline & Risco de sobrepeso & 17 & 23,6 \\
\hline
\end{tabular}

* Dados maternos; ${ }^{* *}$ AM: Aleitamento materno. 
Ao relacionarmos o AM com o estado nutricional, não constatamos associação estatisticamente significativa $(p=0,076)$. Entretanto, verificamos que as crianças que receberam AM por um período inferior a 6 meses, apresentaram prevalência de eutrofia (51\%), seguido de excesso de peso (28,6\%), risco de sobrepeso $(18,4 \%)$ e $2 \%$ apresentaram magreza. Já as crianças que tiveram acesso ao leite materno, entre seis meses a doze meses, também estavam eutróficas $(52,9 \%)$, seguido de risco de sobrepeso $(23,5 \%)$, excesso de peso $(11,8 \%)$ e magreza com $11,8 \%$. Em relação às crianças que não obtiveram o privilégio da amamentação, a maioria apresentou risco de sobrepeso (66,7\%), seguido de eutrofia $(16,7 \%)$ e excesso de peso $(16,7 \%)$.

Na Tabela 2, ao avaliar às práticas alimentares das crianças menores de dois anos, verificou que a maioria consome os mesmos alimentos que a família no jantar e apenas $26,1 \%$ recebeu alimentação da família no dia anterior e 17,4\% recebeu algum tipo de açúcar antes dos 6 meses. Já o consumo de suco industrializado, a maioria das crianças ingeriu no último mês e a maioria não recebeu mingau engrossado com farinha no dia anterior ao da pesquisa, conforme Tabela 2.

Tabela 2. Consumo alimentar de crianças <2 anos, matriculadas em uma creche de uma Instituição Hospitalar de Passo Fundo / RS, 2019. ( $\mathrm{n}=23)$

\begin{tabular}{lccc}
\hline Práticas alimentares & Categoria & $\mathbf{n}$ & \% \\
\hline Refeição da família no jantar no dia anterior & Sim & 19 & 82,6 \\
Refeição da família antes dos 6 meses & Não & 4 & 17,4 \\
& Sim & 6 & 26,1 \\
Mel, melado, açúcar antes dos 6 meses & Não & 17 & 73,9 \\
Suco industrializado no último mês & Sim & 4 & 17,4 \\
\multirow{2}{*}{ Mingau ou leite engrossado com farinha no dia anterior } & Não & 19 & 82,6 \\
& Sim & 12 & 52,2 \\
& Não & 11 & 47,8 \\
& Não & 4 & 17,4 \\
\hline
\end{tabular}

Em relação à alimentação das crianças maiores de dois anos, a Tabela 3 demonstra os aspectos positivos da alimentação, sendo que $87,8 \%$ consomem os alimentos da família no jantar e 42,9\% suco natural de frutas ou fruta de três a quatro vezes na semana. Os alimentos industrializados tiveram um percentual mais elevado, a frequência que consomem salgadinhos de pacote e bolacha recheada foi de duas vezes na semana ou menos, já as bebidas que contém açúcar, verificou-se que 44,9\% das crianças consomem todos os dias, dado esse, que merece muita atenção. 
Tabela 3. Consumo alimentar de crianças >2 anos, matriculadas em uma creche de uma Instituição Hospitalar de Passo Fundo / RS, 2019. $(n=49)$

\begin{tabular}{llll}
\hline Alimentos & Frequência & $\mathbf{n}$ & $\%$ \\
\hline Frutas ou suco de frutas fresco & Dia sim, dia não & 21 & 42,9 \\
& Todos os dias & 17 & 34,7 \\
& Às vezes & 10 & 20,4 \\
& Nunca & 1 & 2,0 \\
\hline Salgadinhos de pacote & Às vezes & 41 & 83,7 \\
& Nunca & 6 & 12,2 \\
& Dia sim, dia não & 2 & 4,1 \\
\hline Bolacha recheada & Às vezes & 31 & 63,3 \\
& Dia sim, dia não & 9 & 18,4 \\
& Todos os dias & 6 & 12,2 \\
& Nunca & 3 & 6,1 \\
\hline Sucos, leites, chás e outras & Todos os dias & 22 & 44,9 \\
bebidas com açúcar & Às vezes & 17 & 34,7 \\
& Dia sim, dia não & 8 & 16,3 \\
& Nunca & 2 & 4,1 \\
\hline
\end{tabular}

Na Tabela 4, ao comparar o consumo alimentar das crianças com a faixa etária menor e maior de dois anos, pode-se observar que as crianças com idade menor de dois anos, mantêm uma alimentação mais adequada, um maior percentual de frutas, verduras, legumes e consumo de feijão. As crianças com idade $>$ de 2 anos apresentaram 0 consumo de carne em maior prevalência e ainda relacionada a essa faixa etária, foi constatado que a frequência do consumo de feijão ocorreu em dias alternados em 42,9\% (21), seguido de 38,8\% (19) todos os dias, 12,2\% (6) às vezes e $6,1 \%$ (3) relataram nunca consumir.

Tabela 4. Consumo alimentar de crianças matriculadas em uma Escola de Educação Infantil de Passo Fundo / RS, 2019. (n=72)

\begin{tabular}{lll}
\hline Consumo de alimentos & \multicolumn{2}{c}{ Faixa etária } \\
\cline { 2 - 3 } & $<2$ anos & $>$ 2anos \\
& $\mathbf{n}(\%)$ & $\mathbf{n}(\%)$ \\
\hline Verduras/legumes* $^{*}$ & $20(87)$ & $34(69,4)$ \\
Frutas $^{*}$ & $22(95,7)$ & $39(79,6)$ \\
Carne $^{*}$ & $19(82,6)$ & $46(93,9)$ \\
Feijão** $^{*}$ & $17(73,9)$ & --- \\
\hline
\end{tabular}

* Consumo de alimentos no dia anterior; ** Consumo de acordo com a frequência de consumo (>2 anos). 
Quanto ao consumo de refrigerante, $69,6 \%$ das crianças menores de dois anos não ingeriram o mesmo no último mês, já as crianças maiores de dois anos, 75,5\% têm o hábito de ingerir a bebida, duas vezes na semana.

Em relação ao ato de se alimentar assistindo televisão, verificamos que $67,3 \%$ das crianças com idade superior a dois anos costumam assistir televisão no momento da alimentação, enquanto as crianças com idade inferior a dois anos tiveram um percentual de $65,2 \%$.

\section{DISCUSSÃO}

O Ministério da Saúde recomenda aleitamento materno exclusivo nos primeiros seis meses de vida e a partir desse momento, deve ter continuidade até os dois anos ou mais de idade sendo complementado com outros alimentos ${ }^{11}$.

Os resultados da pesquisa evidenciaram que a maioria não recebeu AME até os 6 meses, sendo que os motivos principais da interrupção precoce foram leite insuficiente e retorno ao trabalho ou estudo, o que assemelha com o estudo de Amaral ${ }^{12}$ em relação ao leite insuficiente, fruto da insegurança materna quanto a sua capacidade de nutrir plenamente a criança, além da falta de conhecimento das nutrizes, assim como a crença de produção insuficiente de leite, dificuldade de pega da mama e diversas intercorrências mamárias que podem surgir no pós-parto associado a falta de confiança e aos conselhos de familiares e amigos.

Em contrapartida, foi observada uma maior escolaridade que, segundo Silva em $2017^{13}$, mulheres com menor grau de escolaridade tendem a desmamar precocemente as crianças, quando comparadas àquelas com maior nível de escolaridade, pois obtiveram menos chances de receber informações sobre os benefícios do AME até o sexto mês. Além disso, os autores também observaram que com a crescente inserção da mulher no mercado de trabalho, pois as mães com menor renda familiar precisam voltar ao trabalho, favorecendo assim a interrupção precoce do AM.

Ao avaliar o tempo de AME, em 30 crianças na faixa etária de 2 meses a 1 ano e 7 meses de idade, Brito em $2018^{14}$ observou que $64 \%$ não mamaram exclusivamente até $06^{\circ}$ mês, o que se assemelha com o presente estudo. Rieth e Coimbra ${ }^{15}$ também encontraram percentual elevado (57\%) de crianças que não receberam AME até o sexto mês, porém a maioria das crianças deste estudo puderam ser amamentadas até 24 meses de idade (57,3\%), uma vez que a OMS recomenda aleitamento materno até dois anos de idade ou mais.

A avaliação do estado nutricional tem-se tornado aspecto cada vez mais importante no estabelecimento de situações de risco, no diagnóstico nutricional e no planejamento de ações de promoção à saúde e prevenção de doenças. Sua importância é reconhecida tanto na atenção primária, para acompanhar o crescimento e a saúde da criança e do adolescente, quanto na detecção precoce de distúrbios nutricionais, seja desnutrição, seja obesidade ${ }^{8}$. Notou-se no presente estudo, que a eutrofia foi prevalente, o que corrobora com estudos realizados em $2015^{16}$ e $2017^{17}$ onde $60 \%$ e $50 \%$ das crianças estavam eutróficas quando avaliadas através do IMC/idade, respectivamente. 
No presente estudo, não foi possível verificar diferença estatisticamente significativa entre as crianças com AM e o estado nutricional. Porém, tanto as crianças que receberam AM por um período inferior a 6 meses, como as que receberam por um período de 6 a 12 meses encontravam-se em eutrofia. Dado semelhante foi encontrado por Santos ${ }^{18}$ que ao avaliar a duração do aleitamento materno e verificar a sua associação com o estado nutricional de 80 crianças observou-se que a maioria, apresentou estado nutricional adequado.

Um estudo publicado em $2011^{19}$, avaliou 134 pré-escolares de 3 a 5 anos de idade, de uma escola particular de Brasília e ressaltou que as crianças com AME até o $6^{\circ}$ mês apresentaram um excesso de peso de 21,2\% e menor ocorrência de sobrepeso e obesidade, enquanto nas com AME até $02^{\circ}$ mês, foi de $26,7 \%$, o que se assemelha com 0 atual estudo.

Nas práticas alimentares, em relação aos aspectos positivos, estudo de Coelho et al., ${ }^{20}$ observaram-se que $82,1 \%$ consumiram frutas, corroborando com o presente estudo. 0 consumo de legumes e verduras, e também de carne, obtiveram um percentual inferior ao comparar com o atual estudo $(74,1 \%)$ e $(78,5 \%)$, respectivamente. Já o consumo de feijão foi de 76,1\%, sendo que no presente estudo, encontrou-se um valor inferior ao estudo analisado (73,9\%).

Na dieta infantil, as frutas são recomendadas pelo Ministério da Saúde, pois são alimentos ricos em vitaminas, minerais e fibras, e seu consumo auxilia na prevenção e no controle da obesidade. Da mesma forma que, indiretamente, previne contra outras doenças crônicas não-transmissíveis, dentre elas, (diabetes, doenças cardíacas e alguns tipos de câncer), cujo risco é aumentado pela obesidade ${ }^{21}$.

Num estudo de Bortolini, Gubert e Santos², intitulado como "Consumo alimentar de crianças brasileiras com idade de 6 a 59 meses", encontram-se os percentuais mais elevados de consumo diário em legumes, frutas, verduras e carnes nas crianças residentes no Sul, se comparados a outros estados do Brasil, porém a porcentagem que mais consumia doces e refrigerantes também manteve-se no Sul do Brasil.

Quanto à ingestão de sucos industrializados, Coelho et al., ${ }^{20}$ verificou que $98,7 \%$ das crianças avaliadas apresentaram consumo inadequado em relação às recomendações para a faixa etária, sendo maior frequência de inadequação para o consumo de suco ou refresco industrializado no último mês $(60,8 \%)$, assemelhando-se ao do presente estudo.

Diante desse fato, o Ministério da Saúde sugere que na prática do aleitamento materno, o suco e outras bebidas não devem ser oferecidos antes dos seis meses de idade. Esse fato diferencia o atual estudo do estudo publicado por Silva ${ }^{23}$, em que mais da metade das crianças avaliadas, tiveram oferta de refrigerantes ou suco industrializado até o final do primeiro ano de vida.

Ao compararmos o consumo alimentar de crianças $<2$ anos e $>2$ anos, constatamos que crianças com idade menor de dois anos mantêm uma alimentação mais adequada, o que difere de estudo de Oliveira ${ }^{24}$. Neste estudo foi observado que as crianças de 6 a 23 meses apresentaram prevalência de consumo abaixo das recomendações para 
os grupos de frutas e carnes. Porém, no mesmo estudo, crianças maiores de dois anos tiveram ingestão superior às recomendações, fato justificado devido às crianças maiores, participarem dos hábitos alimentares da família o que acaba influenciando o comportamento alimentar.

Dessa forma, Bertuol e Navarro ${ }^{16}$ salientam que a obesidade infantil vem sendo um assunto preocupante na nossa sociedade, visto que os números vêm crescendo cada vez mais entre pré-escolares e escolares. Isso ocorre devido à alteração dos padrões alimentares, como a diminuição da ingestão de frutas e verduras e o aumento do consumo de ultraprocessados e de refrigerantes tem contribuído para o aumento da obesidade.

Por outro lado, há também a grande preocupação em relação ao hábito de se alimentar assistindo televisão. Estudos verificaram que $60 \%$ e $52 \%$ das crianças avaliadas alimentam-se assistindo televisão, respectivamente ${ }^{25,26}$, diferindo do presente estudo que constatou um percentual mais elevado $(65,2 \%)$ de crianças que possuem esse hábito.

\section{CONSIDERAÇÕES FINAIS}

Diante dos resultados, observou-se neste estudo que a maioria das crianças não recebeu AM até o sexto mês de vida, conforme recomendação do MS. Mesmo com prevalência de eutrofia, segundo IMC/idade, foi constatado que 0 consumo alimentar das crianças maiores de dois anos em sua maioria encontra-se em risco para futuras complicações quando comparado a menores de dois anos. No entanto, foi identificado um fator positivo pelo consumo de frutas, verduras, legumes, carnes e feijão, em ambas as idades.

Dessa forma, torna-se fundamental incentivar a alimentação saudável, pois ela tem influência nas características do crescimento, desenvolvimento infantil e intelectual da criança, além de destacar que a influência dos pais em relação à alimentação dos seus filhos deve ser positiva, bem como no ambiente escolar, para que possam atuar como agentes transformadores nos hábitos alimentares das crianças.

\section{REFERÊNCIAS}

1. Rocha, LB; Araujo, FMS; Rocha, NCO; Almeida, CD; Santos, MO; Rocha, CHR. Aleitamento materno na primeira hora de vida: uma revisão de literatura. Rev. Med. Saúde, Brasília, 2017;6(3): 384-394

2. World Health Organization. Defining the problem of overweight and obesity. In: World Health Organization. Obesity: preventing and managing the global epidemic: report of a Who Consultation (WHO Technical Report Series, 894). Geneva: WHO; 2000. p. 241-3. 
3. Oliveira, AF. et al. Avaliação Nutricional da Criança e do Adolescente: Manual de Orientação. Rio de janeiro: Departamento de Nutrologia Sociedade Brasileira de Pediatria, 2009. 45p.

4. Sombra, PV et al. Alimentação complementar e ingestão de alimentos industrializados em crianças menores de três anos. Saúde e Desenvolvimento Humano, Centro Universitário La Salle - UNILASALLE, 2017;5(3):45-51.

5. Lourenço, ADSN et al. Fatores associados ao ganho de peso rápido em pré-escolares frequentadores de creches públicas. Revista Paulista de Pediatria, 2018;36:292-300.

6. Barros MP. A Influência da Publicidade de Alimentos na Obesidade Infantil: 2015. 41 f. Tese - Curso de Comunicação Social, Faculdade de Tecnologia e Ciências Sociais Aplicadas, Centro Universitário de Brasília, Brasília, 2015.

7. Pontes, TE et al. Orientação nutricional de crianças e adolescentes e os novos padrões e consumo: propagandas, embalagens e rótulos. Revista Paulista de Pediatria, 2009; 27(1):99-105.

8. Oliveira, AF. et al. Avaliação Nutricional da Criança e do Adolescente: Manual de Orientação. Rio de janeiro: Departamento de Nutrologia Sociedade Brasileira de Pediatria, 2009. 45p.

9. Brasil- Ministério da Saúde. Secretaria de Atenção à Saúde. Departamento de atenção básica. Saúde da criança, nutrição infantil. Aleitamento materno e alimentação complementar. Brasília: Ministério da Saúde; 2009.

10. Associação Brasileira de Empresas de Pesquisa (ABEP). Critério de Classificação Econômica Brasil. São Paulo, 2018.

11. Brasil- Ministério da Saúde. Secretaria de Atenção à Saúde. Departamento de atenção básica. Dez passos para uma alimentação saudável: guia alimentar para crianças menores de 2 anos: um guia para o profissional da saúde na atenção básica. Brasília: Ministério da Saúde; 2019.

12. Amaral, JLX et al. Fatores que influenciam na interrupção do aleitamento materno exclusivo em nutrizes. Rev. Gaúcha Enferm. 2015;36(esp):127-34. 
13. Silva, DP; Soares, P; Macedo, MV. Aleitamento materno: causas e consequências do desmame precoce. Revista Unimontes Científica. Montes Claros, 2017; 19(2):147-157.

14. Brito, CT; Silva, JVE; Garcia, LRS. Perfil nutricional de crianças atendidas pelo programa de controle de alergia à proteína do leite de vaca no município de Natal/RN. Revista Humano Ser - UNIFACEX, Natal-RN, 2018; 3(1): 1-18.

15. Rieth, NFA; Coimbra, LC. Caracterização do aleitamento materno em São Luís, Maranhão. Rev. Pesq. Saúde, 2016; 17(1):7-12.

16. Bertuol, CD; Navarro, AC. Consumo alimentar e prevalência de sobrepeso/obesidade em pré-escolares de uma escola infantil pública. Revista Brasileira de Obesidade, Nutrição e Emagrecimento, São Paulo, 2015; 9(52):127-134.

17. Madureira, BM; Santos, GA; Padilha, SS; Faria, NC. Avaliação nutricional de pré-escolares de uma escola da cidade de Sete Lagoas - MG. Revista Brasileira de Ciências da Vida, 2017;4(1).

18. Santos, AJAO; Bispo, AJB; Cruz, LD. Padrão de aleitamento e estado nutricional de crianças até os seis meses de idade. HU Revista, Juiz de Fora, 2016;42(2):119-124.

19. Moraes, JFVN; Giugliano, R. Aleitamento materno exclusivo e adiposidade. Rev Paulista Pediatria 2011;29:152-156.

20. Coelho, LC; Asakura, L; Sachs, A; Erbert, I; Novaes, CRL; Gimeno, SGA. Sistema de Vigilância Alimentar e Nutricional/SISVAN: conhecendo as práticas alimentares de crianças menores de 24 meses. Ciência \& Saúde Coletiva, 2015, 20(3): 727-738

21. Brasil. Ministério da Saúde. Secretaria de Atenção à Saúde. Departamento de atenção básica. Dez passos para uma alimentação saudável: guia alimentar para crianças menores de 2 anos: um guia para o profissional da saúde na atenção básica. Brasília: Ministério da Saúde; 2013.

22. Bortolini, GA; Gubert, MB; Santos, LMP. Consumo alimentar entre crianças brasileiras com idade de 6 a 59 meses. Cad. Saúde Pública, Rio de Janeiro, 28(9):1759-1771, set., 2012. 
23. Silva, GL; Toloni, MHA; Menezes, RCE; Asakura, L; Oliveira, MAA; Taddei, JAAC. Introdução de refrigerantes e sucos industrializados na dieta de lactentes que frequentam creches públicas. Rev. Paul. Pediatr., 2015;33(1):34-41.

24. Oliveira, ESF. Consumo dos grupos alimentares em crianças usuárias da rede pública de saúde do município de Aracaju, Sergipe. Rev. Paul. Pediat., 2012; 30(4):529-536.

25. Rossi CA, Albernaz DO; Vasconcelos, FAG.; Assis, MAA.; Di Pietro, PF. Influência da televisão no consumo alimentar e na obesidade de crianças e adolescentes: uma revisão sistemática. Revista de Nutrição, 2010; 23(4):607-620.

26. Silveira, GLL; Neves, LF; Pinho, L. Fatores associados à alimentação entre crianças atendidas em instituições públicas de educação infantil: estudo transversal, Montes Claros, MG. RASBRAN - Revista da Associação Brasileira de Nutrição. São Paulo, SP, 2017;8(2):20-26. 